PROOFS - PAGE NUMBERS ARE TEMPORARY

JOURNAL OF MECHANICS OF MATERIALS AND STRUCTURES

Vol. , No. , 2019

$11 / 2 \frac{1}{2}$

dx.doi.org/10.2140/jomms.2019...101

\title{
ANALYSIS OF 3D NO-TENSION MASONRY-LIKE WALLS
}

\author{
Deborah Briccola, Matteo Bruggi and Alberto Taliercio
}

\begin{abstract}
Modeling masonry as a linear elastic no-tension material, an original approach is implemented to analyze 3D structural elements, with special attention to walls. Masonry is replaced by a suitable equivalent orthotropic material with spatially varying elastic properties and negligible stiffness in case of tensile strain. An energy-based minimization problem is implemented to define the distribution and the orientation of the equivalent material for a given compatible load, so as to obtain a compressive state of stress throughout the structural element. A regular mesh of hexahedrons is used to speed up the sensitivity analysis. The capabilities of the approach in predicting no-tension stress solutions in masonry walls is shown, considering dead loads and both in-plane and out-of-plane live loads.
\end{abstract}

\section{Introduction}

Masonry, either of stone or brick, is well known to be a composite material with negligible tensile strength, at least orthogonally to the orientation of the mortar joints [Como 2016]. Indeed, most of the models available in the literature to cope with masonry structural elements either incorporate damage Color: 111 laws to describe the progressive microcracking and loss in strength due to tensile stresses (see in particular [Lourenço et al. 1997; Berto et al. 2002; Pelà et al. 2011]), or neglect tensile strength completely [Del Piero 1989; Cuomo and Ventura 2000; Angelillo et al. 2010]. The former family of models provides an effective prediction of the real mechanical response of masonry elements beyond the elastic range. Unfortunately, the determination of the several parameters that define these models is generally a difficult task [Lourenço 2001].

The use of a no-tension material model is apparently very appealing, mainly because it avoids uncertainties related to the estimation of the material tensile strength. Additionally, it is based on a conservative assumption. The no-tension model allows the structural behavior to be evaluated assuming the stress tensor to be negative semidefinite and to depend linearly upon the elastic part of the strain; see e.g., [Benvenuto 1991]. Notwithstanding the apparent simplicity of the no-tension model, the expected discontinuities in the stress and displacement fields give rise to several numerical issues. An overview of the numerical difficulties related to the inherent nonlinearity of the problem can be found, e.g., in [Cuomo and Ventura 2000; Alfano et al. 2000; Marfia and Sacco 2005], where procedures to overcome these issues are investigated as well.

Alternatively, the no-tension assumption can be robustly handled through the minimization of a suitable form of the elastic strain energy. A numerical method was originally proposed in [Angelillo et al. 2010] that seeks the equilibrium of two-dimensional no-tension bodies through the minimization of

The authors gratefully acknowledge the financial support of Fondazione Cariplo through grant number 2017-0317 and Italian Ministry of Education University and Research through grant number 2015JW9NJT..

Keywords: no-tension materials, masonry walls, pushover analysis. 
$1 \frac{1}{2} \frac{1}{2}$

3

4

5

6

7

8

9

10

11

12

13

14

15

$\frac{15}{16}$

$\frac{16}{17}$

$\frac{17}{18}$

18

19

$20^{1} / 2$

21

22

the potential energy. Following this approach, an equivalent optimization problem was formulated in [Bruggi 2014] and implemented in [Bruggi and Taliercio 2015] to investigate the in-plane and out-ofplane behavior of masonry walls through simplified 2D models.

Limit analysis can also be applied to evaluate the collapse load and the relevant failure mechanism(s) of no-tension masonry walls. According to Heyman [1966],

(i) the tensile strength is assumed to vanish,

(ii) the "tensile strains" are assumed to be unbounded, and

(iii) the compressive strength of masonry is assumed to be unlimited.

The above assumptions match the behavior of a standard ductile material for which classical limit analysis applies. Limit analysis focuses on the incipient collapse (strains in compression are completely neglected in favor of the additional assumption of rigid-block mechanisms), whereas the linear elastic no-tension model can handle both serviceability and incipient collapse.

This contribution extends the formulation originally presented in [Bruggi 2014] for two-dimensional problems to three-dimensional ones, with special attention to masonry walls. The real no-tension material is replaced by an equivalent orthotropic material, exhibiting negligible stiffness in any direction along which a tensile strain arises. The elastic constants of the equivalent material along its symmetry axes are reduced with respect to those of the real material using a smooth penalization law. For any given load that is compatible with the no-tension assumption, the equilibrium of the body is sought by minimizing the strain energy with respect to the distribution of the equivalent orthotropic material. The proposed algorithm is implemented using displacement-based finite elements and methods of sequential convex programming. A regular mesh of eight node displacement-based finite elements is used to speed up the sensitivity analysis required by the minimization algorithm.

The capabilities of the approach in predicting no-tension stress solutions in masonry walls is shown by addressing both in-plane and out-of-plane loads. The collapse load of walls subject to horizontal actions is predicted by running a sequence of independent analyses on the same discrete model.

The layout of the paper is as follows. The mathematical formulation of the problem governing the analysis of no-tension bodies is described in Section 2: the properties of the equivalent orthotropic material are defined in Section 2A, whereas the energy-based approach employed to distribute and determine the directions of the equivalent orthotropic material is outlined in Section 2B. To assess the capabilities of the proposed approach in the simulation of the structural response of three-dimensional no-tension structures, four case studies are presented in Section 3. Finally, the main results of the work are summarized and ongoing developments of the research are outlined in Section 4.

\section{Problem formulation}

2A. Equivalent orthotropic material. A 3D solid made of an isotropic linear elastic masonry-like material occupies a domain $\Omega$. A triplet of orthogonal Cartesian (global) coordinates, $z_{1}, z_{2}, z_{3}$, defines the position of any point $\chi \in \Omega$. The material does not support tension, meaning that the stress tensor $\sigma$ is negative semidefinite. The infinitesimal strain tensor $\epsilon$ is assumed to be the sum of an elastic part $\epsilon^{e}$, related to the stress $\sigma$ through a linear constitutive tensor, and a "latent" part $\epsilon^{c}$, a positive semidefinite strain that is orthogonal to $\sigma$ and accounts for cracks. The above material model differs from the Heyman 
$1 \frac{1}{2} \frac{1}{2}$

3

4

5

6

7

8

$\overline{9}$

10

11

12

13

14

15

$\frac{15}{16}$

$\frac{17}{18}$

$\frac{18}{19}$

$20^{1} / 2 \frac{20}{21}$

$\frac{21}{22}$

$\frac{\frac{22}{23}}{\frac{24}{25}} \quad \widetilde{\boldsymbol{C}}=\left[\begin{array}{cccccc}1 / \widetilde{E}_{1} & -\tilde{v}_{21} / \widetilde{E}_{2}-\tilde{v}_{31} / \widetilde{E}_{3} & 0 & 0 & 0 \\ -\tilde{v}_{12} / \widetilde{E}_{1} & 1 / \widetilde{E}_{2} & -\tilde{v}_{32} / \widetilde{E}_{3} & 0 & 0 & 0 \\ -\tilde{v}_{13} / \widetilde{E}_{1}-\tilde{v}_{23} / \widetilde{E}_{2} & 1 / \widetilde{E}_{3} & 0 & 0 & 0 \\ 0 & 0 & 0 & 1 / \widetilde{G}_{12} & 0 & 0 \\ 0 & 0 & 0 & 0 & 1 / \widetilde{G}_{23} & 0 \\ 0 & 0 & 0 & 0 & 0 & 1 / \widetilde{G}_{31}\end{array}\right]$.

In (2-2), $\widetilde{E}_{i}(i=1,2,3)$ is the Young's modulus of the equivalent material along the symmetry axis $\tilde{z}_{i}, \widetilde{G}_{i j}$ $(i, j=1,2,3)$ is the shear modulus in the symmetry plane $\left(\tilde{z}_{i}, \tilde{z}_{j}\right)$ and $\tilde{v}_{i j}(i, j=1,2,3)$ is the Poisson's ratio along $\tilde{z}_{j}$ under uniaxial tension along $\tilde{z}_{i}$. The equalities $\tilde{v}_{12} / \widetilde{E}_{1}=\tilde{v}_{21} / \widetilde{E}_{2}, \tilde{v}_{13} / \widetilde{E}_{1}=\tilde{v}_{31} / \widetilde{E}_{3}$, and $\tilde{v}_{23} / \widetilde{E}_{2}=\tilde{v}_{32} / \widetilde{E}_{32}$ hold.

The elastic properties of the equivalent orthotropic material along its symmetry axes are assumed to be governed by three nondimensional fields $\rho_{i}(i=1,2,3)$ whose values range between $\rho_{\min }(>0)$ and 1 , as

$$
\widetilde{E}_{i}=\rho_{i}^{p} E, \quad \tilde{v}_{i j}=\sqrt{\rho_{i}^{p} / \rho_{j}^{p}} \nu, \quad \widetilde{G}_{i j}=\sqrt{\rho_{i}^{p} \rho_{j}^{p}} G \quad(i, j=1,2,3),
$$

where $E, v$, and $G=E /(2(1+v))$ are the Young's modulus, the Poisson's ratio and the shear modulus of the isotropic material, respectively, and $p$ is a penalization parameter (taken equal to 3; see, e.g., [Bendsøe and Sigmund 1999]). $\rho_{i}(i=1,2,3)$ can be seen as "normalized material densities" along $\tilde{z}_{i}$. The interpolation in (2-3) is conceived so as to provide vanishing stiffness in any direction along which a variable attains its minimum value, while full stiffness is preserved in any direction along which a variable 


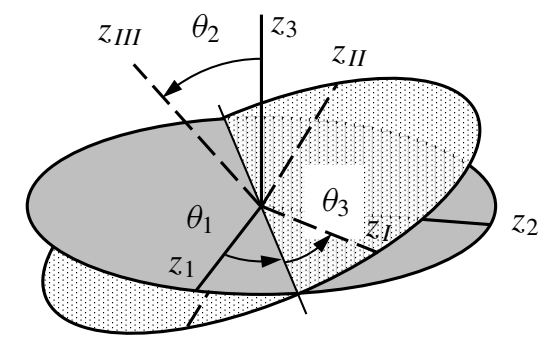

Figure 1. Euler's angles defining the orientation of the symmetry axes of the equivalent orthotropic material to the global reference system.

attains its maximum value. This allows the stress-dependent elastic properties of the isotropic no-tension material to be matched; see also the anisotropic damage law proposed in [Papa and Taliercio 2005]. A strictly positive lower bound, $\rho_{\min }$, is needed to avoid any singularity of the compliance tensor $\widetilde{\boldsymbol{C}}$. This is needed in order to ensure that the stiffness matrix of the body $\boldsymbol{K}$ is positive definite when using a finite element discretization to solve the elasticity equations for any set of normalized material densities. In view of the adoption of optimization methods, normalized material densities are formulated as continuous variables, instead of discrete ones, to exploit efficient methods of mathematical programming for large scale problems; see Section 2B.

Finally, denoting by $\sigma$ and $\epsilon$ the arrays of the stress and strain components in the global Cartesian reference system $O z_{1} z_{2} z_{3}$, the direct form of the stress-strain law in this system can be written as

$$
\boldsymbol{\sigma}=\boldsymbol{D}\left(\rho_{1}, \rho_{2}, \rho_{3} ; \theta_{1}, \theta_{2}, \theta_{3}\right) \boldsymbol{\epsilon},
$$

where $\theta_{i}(i=1,2,3)$ are the Euler's angles that provide the orientation of the symmetry axes of the equivalent orthotropic material with respect to the global Cartesian reference system as represented in Figure 1 (see, e.g., [Rovati and Taliercio 2003]) and

$$
\boldsymbol{D}=\boldsymbol{q} \widetilde{\boldsymbol{D}} \boldsymbol{q}^{T}
$$

In (2-5) the stiffness tensor $\widetilde{\boldsymbol{D}}=\widetilde{\boldsymbol{C}}^{-1}$ and $\boldsymbol{q}=\boldsymbol{q}\left(\theta_{1}, \theta_{2}, \theta_{3}\right)$ is a transformation matrix which can be split into four submatrices:

$$
\boldsymbol{q}=\left[\begin{array}{ll}
\boldsymbol{q}_{A A} & \boldsymbol{q}_{A B} \\
\boldsymbol{q}_{B A} & \boldsymbol{q}_{B B}
\end{array}\right]
$$

the entries of the submatrices are given by

$$
\begin{array}{ll}
\left(\boldsymbol{q}_{A A}\right)_{i j}=Q_{i \alpha}^{2}, & \left(\boldsymbol{q}_{A B}\right)_{i j}=\sqrt{2} \in_{\alpha \beta \gamma} Q_{i \beta} Q_{j \gamma}, \\
\left(\boldsymbol{q}_{B A}\right)_{i j}=\sqrt{2} \epsilon_{i h k} Q_{h \alpha} Q_{k \alpha}, & \left(\boldsymbol{q}_{B B}\right)_{i j}=\epsilon_{i h k} \in_{\alpha \beta \gamma}\left(Q_{h \beta} Q_{k \gamma}+Q_{h \gamma} Q_{k \beta}\right) .
\end{array}
$$

In (2-7), $Q_{i \alpha}$, etc., are the director cosines of the principal stress directions (i.e., the material symmetry axes of the equivalent orthotropic material) to the global reference system: $Q_{i \alpha}=\cos \left(z_{i}, z_{\alpha}\right)(i=1,2,3$; $\alpha=I, I I, I I I)$. Summation is implied over any repeated index, and $\alpha=I$ if $j=1, \alpha=I I$ if $j=2$, $\alpha=I I I$ if $j=3$. Unlike the classical alternating symbol, $\epsilon_{i h k}$ and $\epsilon_{\alpha \beta \gamma}$ are equal to 1 if $(i, h, k)$ or $(\alpha, \beta, \gamma)$ are even permutations of $(1,2,3)$ or $(I, I I, I I I)$, respectively, and vanish in any other case (see, e.g., [Cowin and Mehrabadi 1995]). 
2B. Energy-based analysis of no-tension 3D solids. In Section $2 \mathrm{~A}$ an elastic orthotropic material has been defined, which matches the behavior of the no-tension solid through suitable stiffness relaxations provided by the set of normalized material densities. Cracking strains arise along the axes of negligible stiffness (for minimum values of these normalized densities) mainly to restore strain compatibility; see in particular [Angelillo et al. 2010]. This allows the behavior of the no-tension solid to be modeled for any multiaxial stress state using a single material model, with point-wise varying elastic constants. Stress and/or strain discontinuities are matched by abrupt changes in the normalized material density fields.

The equilibrium of any linear elastic no-tension solid can be solved through an energy-based approach as a problem of optimal distribution of material; see [Bruggi 2014] for the 2D rationale. The equivalent orthotropic material defined in Section $2 \mathrm{~A}$ is distributed over the body to minimize the overall strain energy while enforcing nonpositive principal stresses everywhere.

Adopting a displacement-based numerical approach, the continuous formulation of this minimization problem can be stated as follows:

$$
\begin{gathered}
\min _{\rho_{1}, \rho_{2}, \rho_{3}} \mathscr{E}=\frac{1}{2} \int_{\Omega} \boldsymbol{\epsilon}^{T}(\boldsymbol{u}) \boldsymbol{D}\left(\rho_{1}, \rho_{2}, \rho_{3} ; \theta_{1}, \theta_{2}, \theta_{3}\right) \boldsymbol{\epsilon}(\boldsymbol{u}) d \Omega \\
\text { such that } \int_{\Omega} \boldsymbol{\epsilon}^{T}(\boldsymbol{u}) \boldsymbol{D} \boldsymbol{\epsilon}(\boldsymbol{v}) d \Omega=\int_{\Gamma_{t}} \boldsymbol{t}_{0}^{T} \boldsymbol{v} d \Gamma \quad \forall \boldsymbol{v} \in H^{1},\left.\quad \boldsymbol{u}\right|_{\Gamma_{u}}=\boldsymbol{u}_{0}, \\
\theta_{1}, \theta_{2}, \theta_{3} \mid \tilde{z}_{1}=z_{I}, \tilde{z}_{2}=z_{I I}, \tilde{z}_{3}=z_{I I I}, \\
\rho_{1}, \rho_{2}, \rho_{3} \mid \sigma_{I} \leq 0, \sigma_{I I} \leq 0, \sigma_{I I I} \leq 0, \\
\rho_{\min } \leq \rho_{1}, \rho_{2}, \rho_{3} \leq 1 .
\end{gathered}
$$

In the above statement, the minimization unknowns are the fields of the "densities" $\rho_{i}(i=1,2,3)$ whereas the objective function is the elastic strain energy computed through the displacements $\boldsymbol{u}$ over the 3D domain $\Omega . \Gamma_{u}$ stands for the part of the boundary that is subjected to the given traction $t_{0}$, whereas $\Gamma_{t}$ undergoes prescribed displacements $\boldsymbol{u}_{0}$, being $\Gamma=\Gamma_{t} \cup \Gamma_{u}$. Equation (2-8).2 enforces elastic equilibrium in $\Omega$ and along $\Gamma_{t}$ for any set of normalized densities, being the gradients of $\boldsymbol{u}$ and $\boldsymbol{v}$ square integrable; Equation (2-8).3 prescribes alignment of the symmetry axes of the equivalent orthotropic material to the principal stress directions of the no-tension solid; Equation (2-8).4 requires the normalized densities to define a compression-only stress state all over the domain.

A regular mesh of 8-node hexahedrons is adopted in the simulations to speed up the numerical procedure. Being $N$ the number of discrete solid elements, the proposed formulation reads as follows:

$$
\begin{gathered}
\min _{x_{1 e}, x_{2 e}, x_{3 e}} \mathscr{E}=\frac{1}{2} \sum_{e=1}^{N} \boldsymbol{U}_{e}^{T} \boldsymbol{K}_{e}\left(x_{1 e}, x_{2 e}, x_{3 e} ; t_{1 e}, t_{2 e}, t_{3 e}\right) \boldsymbol{U}_{e}, \\
\sum_{e=1}^{N} \boldsymbol{K}_{e}\left(x_{1 e}, x_{2 e}, x_{3 e} ; t_{1 e}, t_{2 e}, t_{3 e}\right) \boldsymbol{U}_{e}=\boldsymbol{f} \\
t_{1 e}, t_{2 e}, t_{3 e} \mid \tilde{z}_{1}=z_{I}, \tilde{z}_{2}=z_{I I}, \tilde{z}_{3}=z_{I I I} \\
x_{1 e}, x_{2 e}, x_{3 e} \mid \sigma_{I e}, \sigma_{I I e}, \sigma_{I I I e} \leq 0 \\
0<x_{\min } \leq x_{1 e}, x_{2 e}, x_{3 e} \leq 1 \\
e=1 \ldots N .
\end{gathered}
$$


PROOFS - PAGE NUMBERS ARE TEMPORARY

106

DEBORAH BRICCOLA, MATTEO BRUGGI AND ALBERTO TALIERCIO

${ }_{1}^{1 / 2} 1$ The objective function is computed over the $N$ hexahedrons using the same element stiffness matrix, $\boldsymbol{K}_{e}$, and the array of the nodal displacements of each element of the regular mesh $\boldsymbol{U}_{e} ; \boldsymbol{f}$ denotes the array gathering the equivalent nodal loads; $t_{1 e}, t_{2 e}, t_{3 e}$ are the Euler's angles defining the orientation of the equivalent material, i.e., the principal stress directions in the no-tension body in the $e$-th element. The three sets of element-wise unknowns $x_{1 e}, x_{2 e}, x_{3 e}$ correspond to the "normalized material densities" along the symmetry axes of the equivalent orthotropic material in any finite element $e$.

Details on the numerical implementation of the problem in (2-9) can be found in [Bruggi 2014]. The extension of the minimization algorithm from two to three dimensions is straightforward. The method of moving asymptotes [Svanberg 1987] is used to handle the minimization. At each iteration, the current value of the objective function $\mathscr{E}$ and its sensitivities with respect to the densities are needed. This simply reads

$$
\frac{\partial \mathscr{E}}{\partial x_{i e}}=-\frac{1}{2} \boldsymbol{U}_{e}^{T} \frac{\partial \boldsymbol{K}_{e}}{\partial x_{i e}} \boldsymbol{U}_{e}
$$

Instead of implementing demanding stress constraints, following [Ananiev 2005] an efficient procedure that penalizes the energy contributions (and the relevant sensitivities) related to any tensile strain is implemented, thus enforcing a compression-only stress regime. The adoption of a regular mesh of finite elements speeds up remarkably the computation of the quantities in (2-10).

By repeatedly calling the minimization algorithm for different values of the live loads, the collapse load of the structural wall can also be estimated as the value beyond which convergence is lost.

As already mentioned in Section 1, an energy-based approach to the analysis of linear elastic masonrylike materials was presented in [Angelillo et al. 2010] using displacements as unknowns and the total potential energy as the objective function. The elastic problem with unilateral stress constraints was successfully solved through descent methods, by formulating an efficient unconstrained minimization of a convex function.

Alternatively, the energy-based approach proposed in [Bruggi 2014], here extended to three-dimensional bodies, relies on most common methods and techniques used in structural optimization. Normalized material densities govern the stiffness of the equivalent orthotropic material, i.e., the strain energy in the no-tension material, depending on the stress state; see Equation (2-8).4. This allows the equilibrium of linear elastic no-tension bodies to be solved by means of algorithms that were originally conceived to solve large scale problems in structural optimization. The computational cost of the proposed approach is investigated in Section 3 with reference to Example 3.4.

\section{Numerical applications}

In the last decades, finite element approaches for no-tension materials have been extensively adopted to predict collapse multipliers of structural elements as an alternative to limit analysis [Fortunato et al. 2016; 2018; Angelillo et al. 2010; Bruggi and Taliercio 2015]. Four preliminary examples are considered to assess the numerical method presented above, considering vertical dead loads and horizontal live forces. The first one addresses the in-plane collapse mechanism of a reference wall that was previously investigated, among others, in [Maier and Nappi 1990; Bruggi 2014]; the second one focuses on a typical out-of-plane mechanism of a portion of a wall between contiguous stories, which was already investigated in [Bruggi and Taliercio 2015] using a 2D model and the rigid no-tension assumption. The third example 

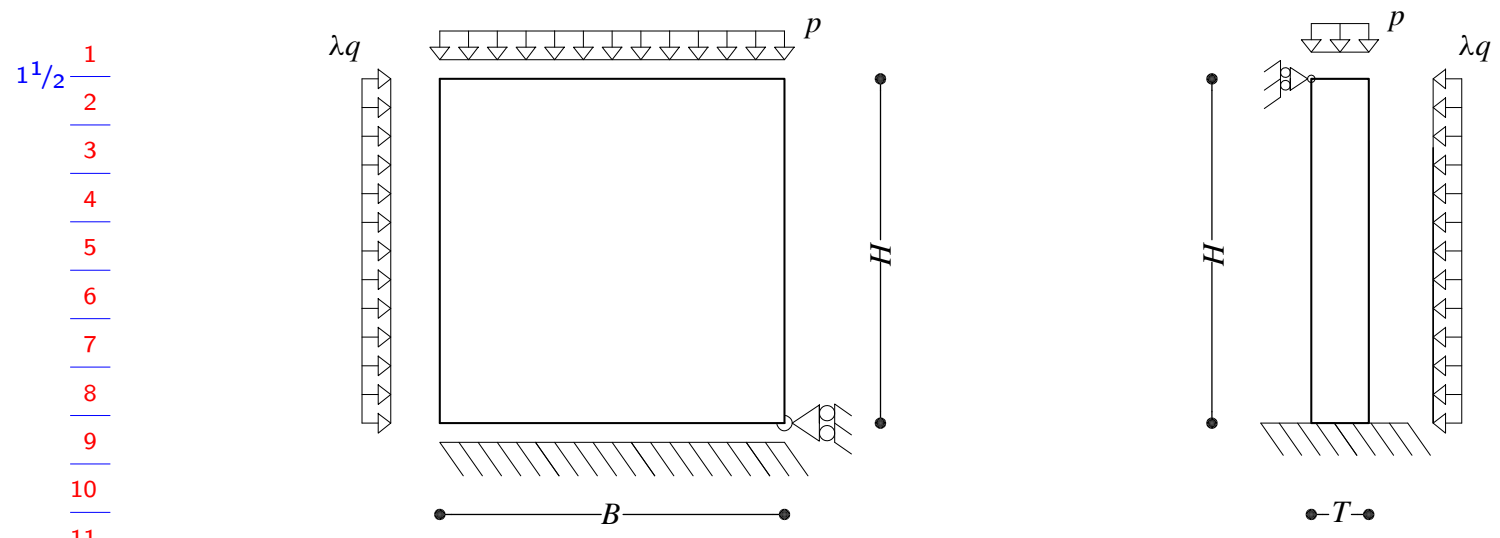

Figure 2. Geometry and boundary conditions for the Examples 3.1 (left) and 3.2 (right).

is a variation of the latter one, which fully exploits the capabilities of the proposed approach to solve the no-tension equilibrium of a three-dimensional solid with any boundary conditions. Finally, the fourth example analyzes the effect of a ground settlement below two intersecting walls.

To enforce consistent loads, i.e., loads for which a no-tension solution is feasible, in all the examples hereafter a compressive prestress equal to $0.01 \mathrm{MPa}$ is applied all over the vertical sides of the walls.

Walls made of no-tension material are taken into account. Referring to Example 3.2, the results given $20^{1} / 2 \frac{20}{21}$ by a conventional linear elastic material model, with symmetric behavior in tension and compression, are also shown to highlight the dramatic difference with respect to those given by the no-tension model.

Plots showing the principal stress directions at the center of each finite element are reported in this section. In these figures, blue stands for compression, whereas red stands for tension. The length of the lines is proportional to the value of the relevant principal stress, and is scaled by the maximum stress represented in each plot.

Example 3.1. A square panel similar to that dealt with in [Maier and Nappi 1990] and investigated, among others, in [Fuschi et al. 1995; Bruggi 2014], is considered. The width and height of the panel are $B=H=1 \mathrm{~m}$, and the thickness $T=0.25 \mathrm{~m}$. A side view (longitudinal) is depicted in Figure 2 (left). The panel is simply supported at the bottom, and is horizontally constrained along the right edge of the base. The Young's modulus is assumed equal to $1000 \mathrm{MPa}$, while the Poisson ratio $v=0.2$. A uniform vertical dead load $p$ of $72 \mathrm{kN} / \mathrm{m}^{2}$ is applied along the top side. A horizontal live load $\lambda q$ acts on the left side along the longitudinal direction, being $q=40 \mathrm{kN} / \mathrm{m}^{2}$ and $\lambda$ the load multiplier.

The behavior of the panel for increasing horizontal loads can be captured effectively by a curve representing the displacement of a control point (herein the top right corner) versus the load multiplier. In Figure 3, the solid line represents the outcome of the numerical simulations originally performed in [Maier and Nappi 1990] adopting an incremental approach over a mesh of $8 \times 8=64$ square elements, whereas dots stand for results achieved by the proposed energy-based procedure using a discretization of $8 \times 8 \times 2=128$ cubic elements. It must be remarked that, for each prescribed value of the load multiplier, the formulation in (2-9) allows the relevant displacement of the control point to be evaluated independently of the previous history. Indeed, simulations were performed for a limited set of multipliers, i.e., $0,0.5,1,1.5,1.7,1.8,1.85,1.9,1.95,2$. The last converged simulation was at $\lambda=1.95$. 


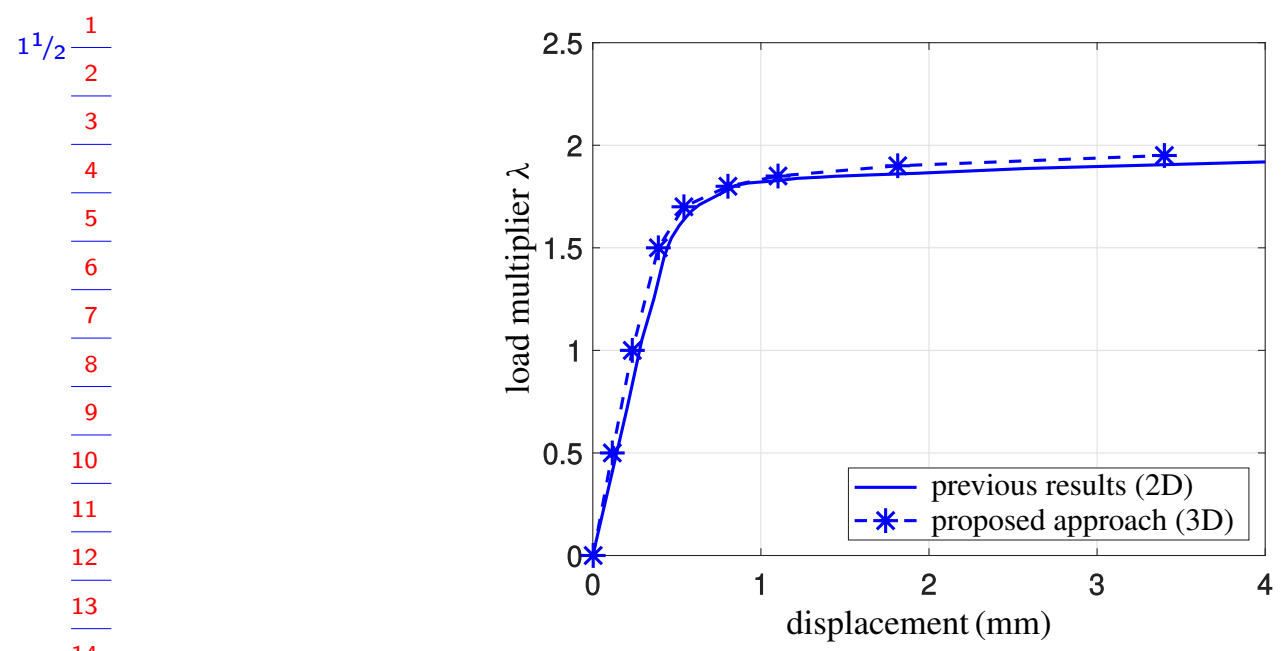

Figure 3. Example 3.1. Top right horizontal displacement of the panel vs. load multiplier $\lambda$. Previous results are from [Maier and Nappi 1990].

Referring to $\lambda=1$, Figure 4 (left) shows the computed distribution of linear isotropic material and nonlinear orthotropic material in the panel. White stands for zones in compression where no inelastic strain is expected $\left(x_{1 e}=x_{2 e}=x_{3 e}=1\right)$ and the panel has full stiffness. Black stands for regions where some inelastic strain arises (at least one of the three unknowns is equal to $x_{\min }$ ) and cracks weaken the panel. Figure 5 (top) shows two views of the relevant map of the principal compressive stresses (blue lines). Vertical compressive reactions are allowed along the base, except for the very first two elements where cracking strains arise. The effect on the overall deformability of the structural element is negligible; see the relevant displacement in Figure 3.

Figure 4 (right) shows the computed distribution of linear isotropic material and nonlinear orthotropic
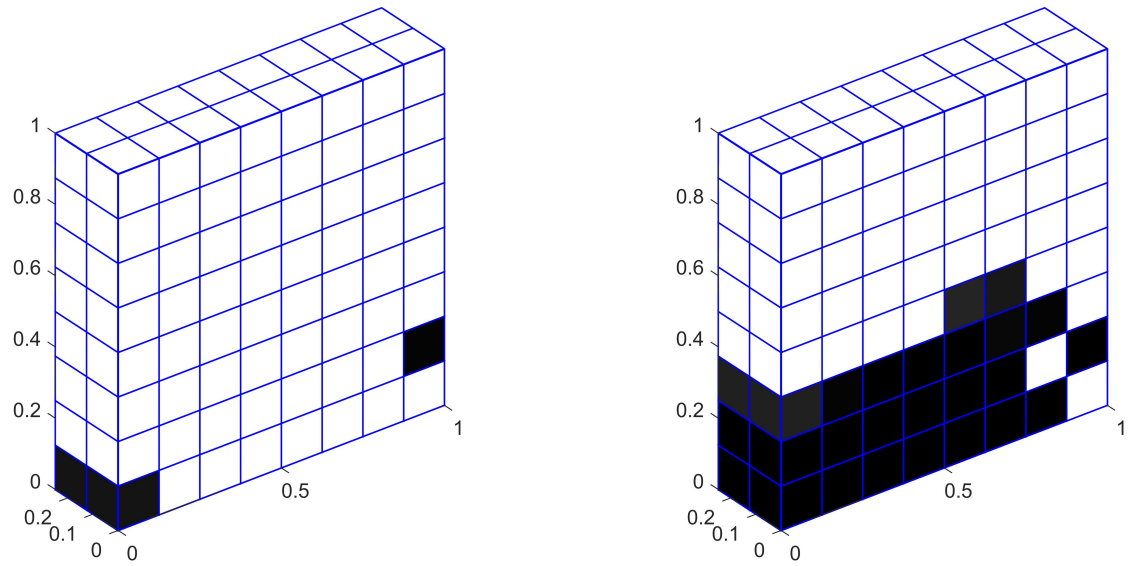

Figure 4. Example 3.1. Distribution of linear isotropic material (white) and nonlinear orthotropic material (black) at $\lambda=1$ (left) and $\lambda=1.95$ (right). 

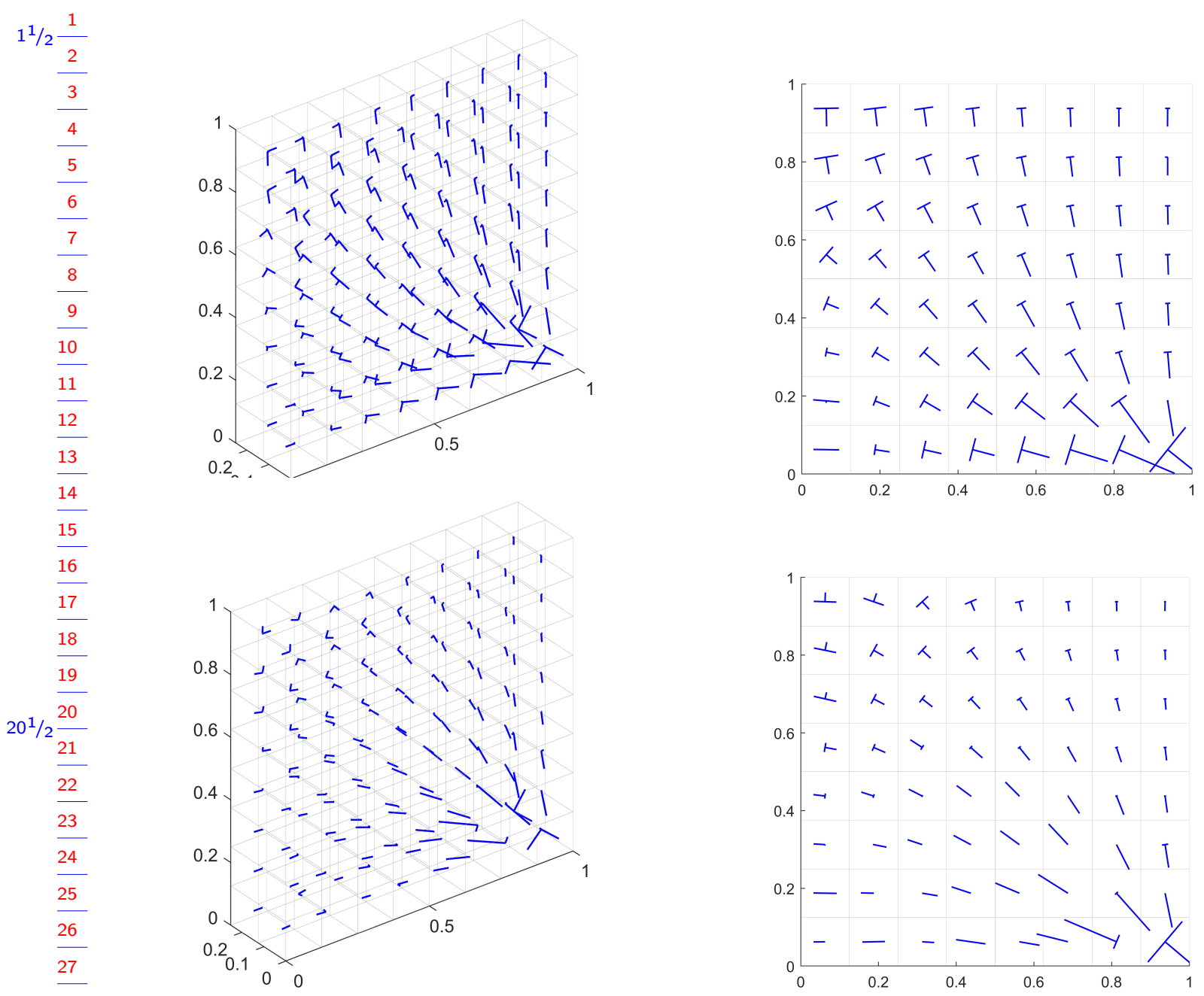

Figure 5. Example 3.1. Principal compressive stresses at $\lambda=1$ (top) and $\lambda=1.95$ (bottom).

material at $\lambda=1.95$, whereas Figure 5 (bottom) shows two views of the relevant map of the principal compressive stresses. Apart from the region in the vicinity of the ground hinge, compressive stresses at the base of the panel are mainly horizontal, whereas an extended cracked zone has a remarkable effect in terms of deformability of the structural element. The global collapse of the structure is incipient. Indeed, no convergence of the proposed minimization procedure can be found at higher values of the load multiplier.

$39^{1 / 2}$ Example 3.2. The second example deals with a rectangular panel of width $B=0.5 \mathrm{~m}$, height $H=1 \mathrm{~m}$, and thickness $T=0.25 \mathrm{~m}$. A side view (lateral) is depicted in Figure 2 (right). The panel is made of a no-tension material having the same mechanical properties used in the previous example. A uniform vertical dead load $p=120 \mathrm{kN} / \mathrm{m}^{2}$ is applied at the top. A horizontal live load $\lambda q$ acts on the left side 

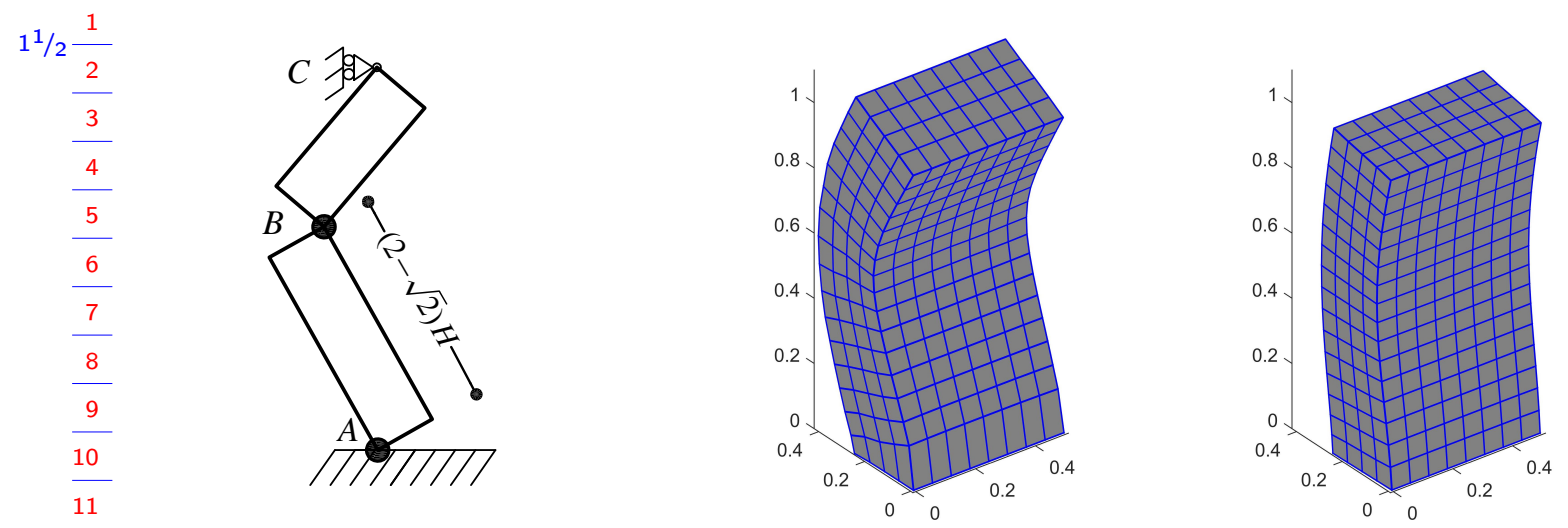

Figure 6. Example 3.2. Theoretical collapse mechanism (left) and magnified displacements at $\lambda=2.1$ for no-tension material (middle) and conventional material (right).

along the transversal direction, being $q=20 \mathrm{kN} / \mathrm{m}^{2}$ and $\lambda$ the load multiplier. The panel is pinned at the base. Transversal displacements are constrained also at the top of the wall.

A similar geometry has been used in [Bruggi 2014] to investigate the out-of-plane behavior of a reference section of a masonry wall under seismic actions, relying on a two-dimensional modeling. Assuming a three-hinge mechanism (see Figure 6 (left)) limit analysis can be straightforwardly applied to compute both the location of the central hinge in the collapse mode, $y_{h}=(2-\sqrt{2}) H$, and the relevant multiplier

$$
\lambda_{c}=\frac{p T^{2}}{q H^{2}}(2 \sqrt{2}+3) .
$$

With the values of the geometrical parameters detailed above, one gets $y_{h}=0.59 \mathrm{~m}$ and $\lambda_{c}=2.18$.

A limited set of simulations was performed with the proposed approach at $\lambda=1.9,2,2.1$, and 2.2. The last converged simulation was at $\lambda=2.1$. Pictures presented next refer to this value of the load multiplier, i.e., to the wall on the verge of collapse.

A plot of the magnified displacements at incipient collapse is represented in Figure 6 (middle). Strains are localized at the base of the wall and, approximately, at $0.6 \mathrm{~m}$ from the ground, in good agreement with the position of hinges A and B in Figure 6 (left). At the same load and the same magnification factor, Figure 6 (right) shows the displacement map in case of a conventional linear elastic material with symmetric behavior in tension and compression.

Figure 7 (left) shows the principal stresses computed at the estimated collapse multiplier in the notension panel. Two views are shown; the second is a side view of the stress map in a vertical section of the wall.

The flux of compressive stresses found in the lower and in the central region of the panel is confined in a very limited part of the section. Indeed, hinges A and B are expected to activate for any additional infinitesimal increase in the live load.

Stress maps are remarkably different if a conventional material with symmetric behavior in tension and compression is considered in the simulations; see Figure 7 (right). At the same load, compressive regions are much more extended than in the case of a no-tension material. Additionally, it must be 
PROOFS - PAGE NUMBERS ARE TEMPORARY
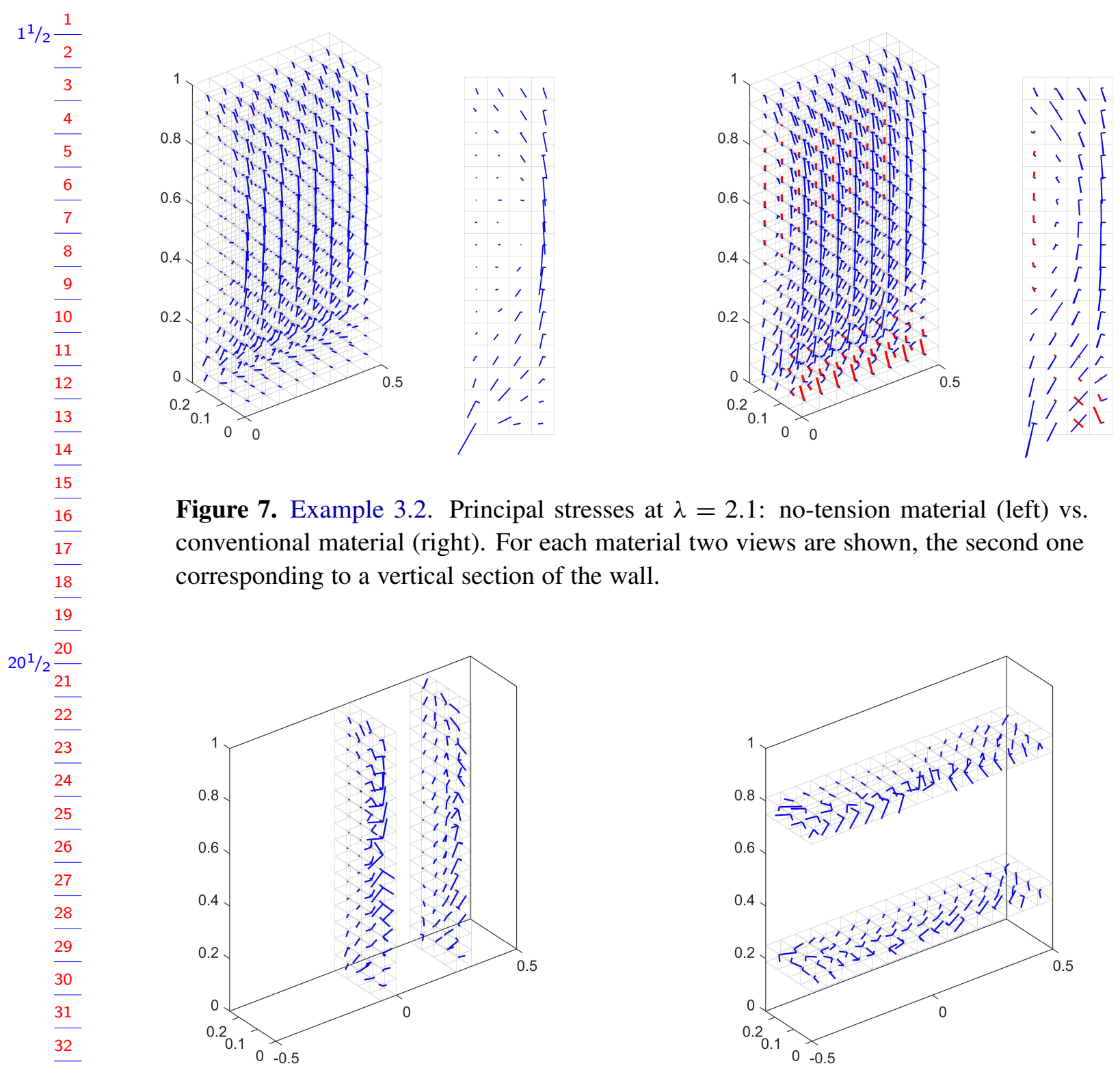

Figure 7. Example 3.2. Principal stresses at $\lambda=2.1$ : no-tension material (left) vs. conventional material (right). For each material two views are shown, the second one corresponding to a vertical section of the wall.

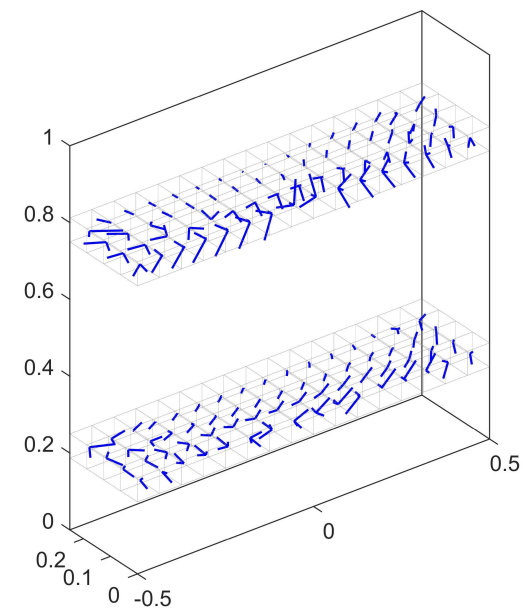

Figure 8. Example 3.3. Principal compressive stresses at $\lambda=4.2$ along representative vertical and horizontal sections.

remarked that the maximum compressive stress computed at the base of the wall for the conventional material is approximately $30 \%$ lower than that found for the no-tension material.

$39^{1} / 2 \frac{39}{40} \quad$ These crucial differences show that the conventional material model should not be used to capture the behavior of masonry structures. The negligible tensile strength and the occurrence of cracks remarkably affect the stress field within the wall. 

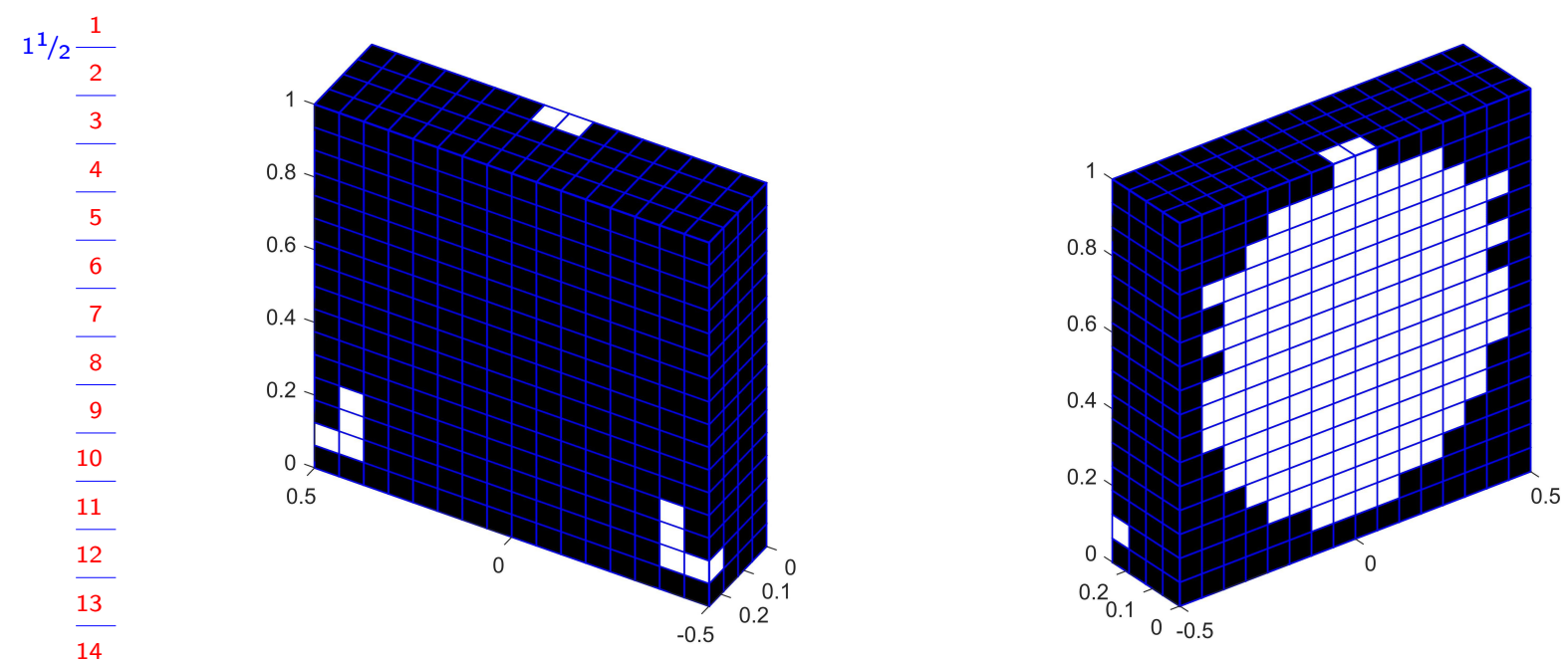

Figure 9. Example 3.3. Distribution of linear isotropic (white) and nonlinear orthotropic (black) material at $\lambda=4.2$.
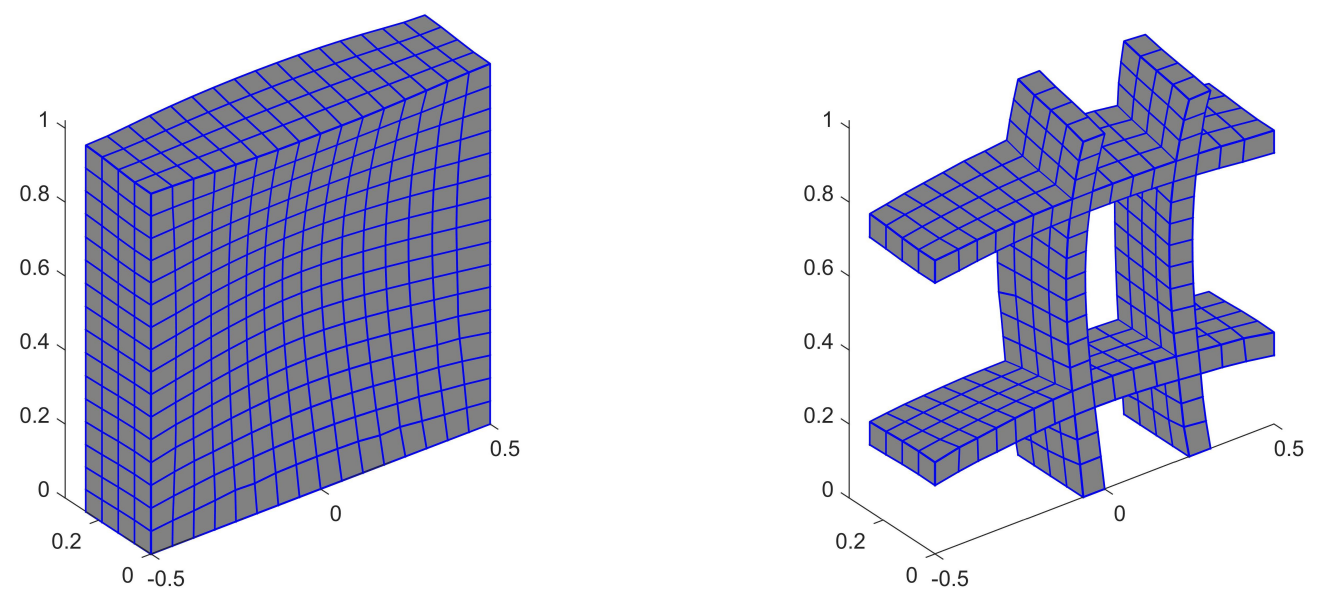

Figure 10. Example 3.3. Magnified displacements at $\lambda=4.2$.

Example 3.3. This example is a variation of the previous one. The material and geometry are the same, apart from the width of the panel that is doubled, i.e., $B=1 \mathrm{~m}$. Indeed, $p=60 \mathrm{kN} / \mathrm{m}^{2}$ and $q=10 \mathrm{kN} / \mathrm{m}^{2}$. Boundary conditions at the bottom and the top are unchanged. Additionally, horizontal displacements are restrained in both the longitudinal and transverse direction all over the shortest vertical sides.

The proposed numerical approach is used to investigate the three-dimensional stress state that arises in the panel made of no-tension material. The case $\lambda=4.2$ is considered.

At each point of the panel, the horizontal load is essentially transferred to the ground by two static schemes that work together. A map of the principal stress directions is shown in Figure 8 addressing 2 two vertical sections and two horizontal sections that are representative of the main load paths. In the 


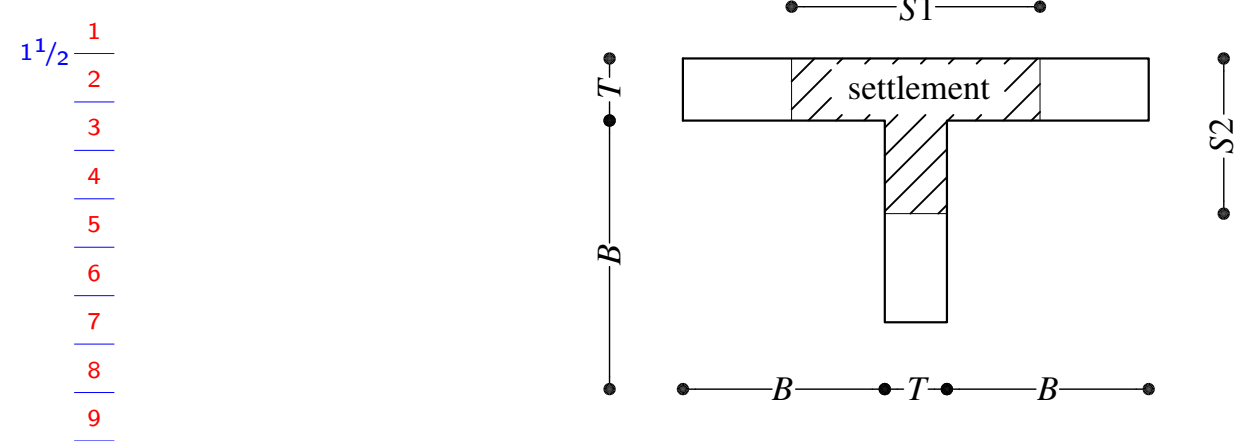

Figure 11. Geometry for Example 3.4.

vertical sections, stresses flow to the top side and the bottom side of the panel in a way similar to Figure 7 (left). In the horizontal sections, stresses flow towards the sides of the panel following effective archlike patterns. Due to the assumption of perfect constraints, an ideal scheme like this one does not give rise to a mechanism; see, e.g., [Como 2016]. Trusts at the lateral springers and allowable compressive stresses can be alternatively controlled to discuss the feasibility of the applied load. Both quantities can be evaluated through the proposed numerical approach.

Figure 9 shows a map of the computed distribution of linear isotropic material and nonlinear orthotropic material in the panel. Cracking strains are expected to weaken the panel in the extended regions marked in black. Figure 10 shows the magnified deformed geometry of the panel (left) and the two sets of representative sections above referred (right). In the loaded side, inelastic strains are mostly located around the constrained boundaries.

Example 3.4. We now consider a T-shaped wall, whose plan view is shown in Figure 11. The wall (of width $B=0.8 \mathrm{~m}$, thickness $T=0.25 \mathrm{~m}$, and height $H=1 \mathrm{~m}$ ) is subject to vertical loads of arbitrary intensity at the top. To simulate the effect of a vertical settlement affecting the connection, the base of the

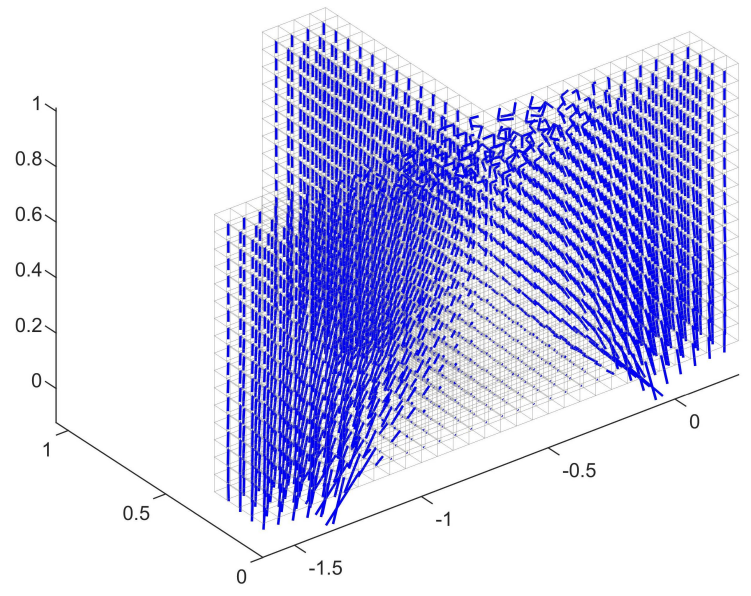

Figure 12. Example 3.4. Principal compressive stresses in case of a vertical settlement: $3 \mathrm{D}$ view. 

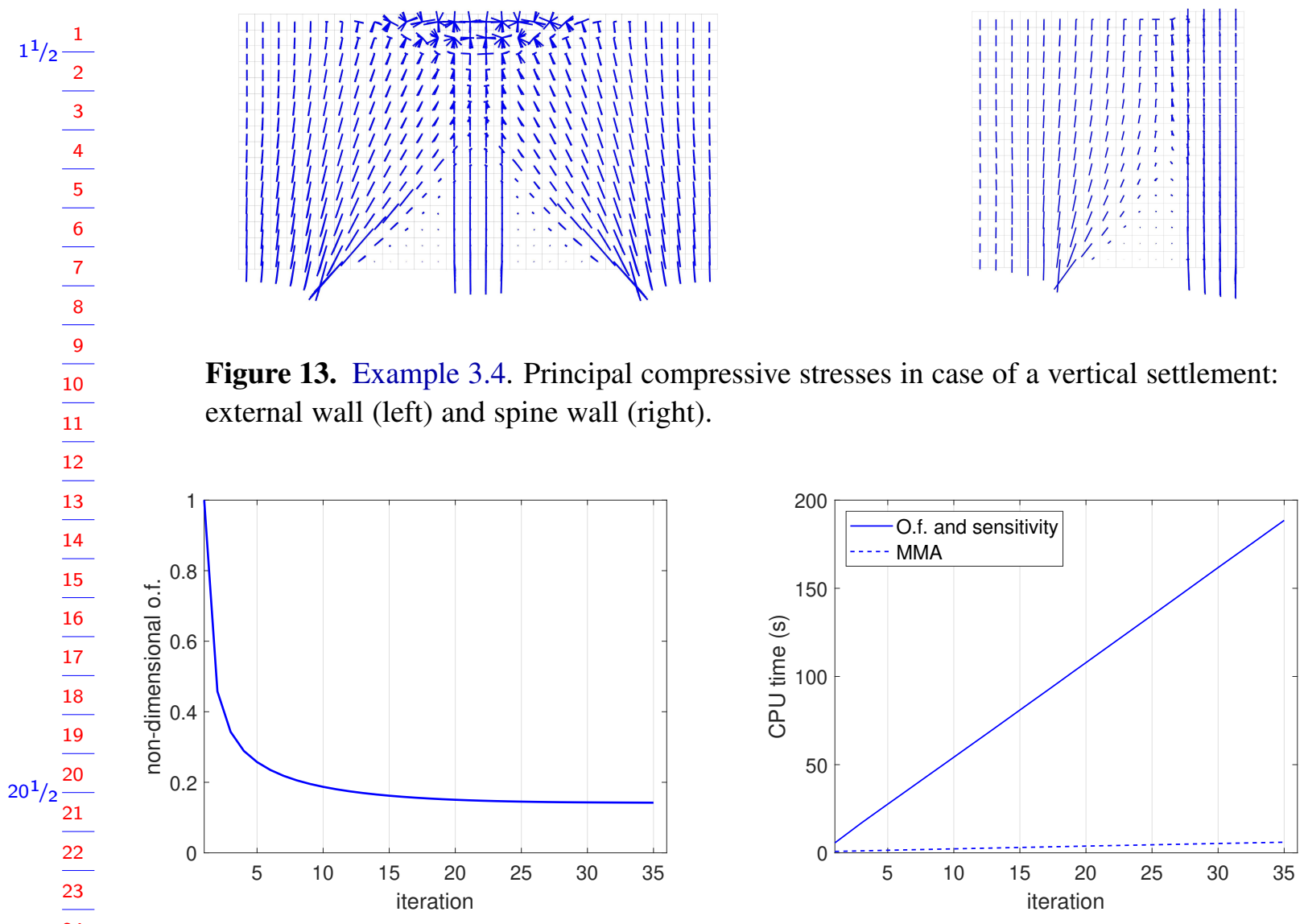

Figure 14. Example 3.4. History plot of the objective function (left) and computational cost (right). Note that o.f. stands for "objective function".

panel is constrained except for the central region with dimension $S 1=1 \mathrm{~m}$ and $S 2=0.625 \mathrm{~m}$, in agreement with the analytical and numerical investigations reported in [Mastrodicasa 1943]. A regular mesh of 2752 elements is used for the simulation, along with 8256 minimization unknowns. Figures 12 and 13 show a map of the principal stresses. An arch-like mechanism arises both in the external wall and in the spine wall transferring the vertical load to the safe external regions; see also Figure 13. The proposed algorithm captures a discontinuity in the stress field that allows a possible detachment in the lower part of the connection to be detected, as shown in [Mastrodicasa 1943]. The nonhomogeneous stress distribution at the base of the external wall suggests that a torsional effect, induced by the vertical settlement, arises.

Figure 14 reports the history plot of the objective function, i.e., the overall strain energy scaled by its value at the first iteration at $x_{1 e}=x_{2 e}=x_{3 e}=0.5$ (left), and the cumulative cost of the proposed algorithm (right), both in terms of time spent in the computation of the objective function and its derivative, and in updating the minimization unknowns through the MMA. Thirty five iterations and approximately 200 seconds are needed on a standard laptop to solve the equilibrium. Most of the time is spent in the finite element analysis (assembly and Cholesky factorization of the stiffness matrix) performed for the current set of minimization unknowns, whereas, as expected, the large scale optimizer is quite efficient. 
$1 / 2 \frac{1}{2}$

3

$\frac{3}{4}$

$\frac{4}{5}$

$\frac{5}{6}$

6

7

8

9

10

11

12

13

14

15

$\frac{15}{16}$

$\frac{16}{17}$

$\frac{17}{18}$

$\frac{18}{19}$

19

$20^{1} / 2 \frac{20}{21}$

$$
\frac{21}{22}
$$

\section{Concluding remarks and future perspectives}

An efficient numerical method has been presented to analyze no-tension 3D structural elements subjected to given loads, according to an energy-based nonincremental algorithm that extends a proposal formulated for 2D bodies [Bruggi 2014]. The occurrence of tensile stresses is prevented by replacing the real material by an equivalent orthotropic material that has negligible stiffness along the direction(s) of the principal tensile strain(s) in the solid. The distribution of material that minimizes the elastic strain energy stored in the body provides the solution of the no-tension equilibrium.

To speed up the computations, the preliminary implementation of the method is based on the adoption of regular meshes of 8-node hexahedral finite elements. When addressing collapse, the relevant mechanism and the load multiplier can be estimated without any a-priori hypothesis regarding the position of the "plastic hinges". For any assigned compatible load and set of boundary conditions, the proposed approach allows the behavior of no-tension solids experiencing triaxial stress states to be investigated.

In the continuation of the work, the simplification adopted so far, according to which only regular meshes of hexahedrons are allowed, will be removed to handle structural elements of general shape, e.g., walls with openings, vaults, and domes.

When acted upon by dynamic loads, masonry constructions are characterized by a lack of energy dissipation with respect to ductile structures. As outlined in [Di Carlo et al. 2017], rocking mechanisms in piers involve only limited regions of the panels, mainly because of diagonal cracking. In principle, the energy-based approach herein proposed could be adopted to solve the steps of a transient dynamic analysis, thus detecting regions that remain ineffective during the development of the mechanism.

Finally, the possibility of defining optimal reinforcing layouts will be dealt with: the proposed formulation can be readily extended to define the distribution of optimal tension-only strengthening layers over the walls, similarly to the approach followed in [Bruggi and Taliercio 2017] for 2D bodies.

\section{References}

[Alfano et al. 2000] G. Alfano, L. Rosati, and N. Valoroso, "A numerical strategy for finite element analysis of no-tension materials", Int. J. Numer. Methods Eng. 48:3 (2000), 317-350.

[Ananiev 2005] S. Ananiev, "On equivalence between optimality criteria and projected gradient methods with application to topology optimization problem”, Multibody Syst. Dyn. 13:1 (2005), 25-38.

[Angelillo et al. 2010] M. Angelillo, L. Cardamone, and A. Fortunato, "A numerical model for masonry-like structures", $J$. Mech. Mater. Struct. 5:4 (2010), 583-615.

[Bendsøe and Sigmund 1999] M. P. Bendsøe and O. Sigmund, "Material interpolation schemes in topology optimization", Arch. Appl. Mech. 69:9-10 (1999), 635-654.

[Benvenuto 1991] E. Benvenuto, An introduction to the history of structural mechanics, II: Vaulted structures and elastic systems, Springer, 1991.

[Berto et al. 2002] L. Berto, A. Saetta, R. Scotta, and R. Vitaliani, "An orthotropic damage model for masonry structures", Int. J. Numer. Methods Eng. 2 (2002), 127-157.

[Bruggi 2014] M. Bruggi, "Finite element analysis of no-tension structures as a topology optimization problem", Struct. Multidiscip. Optim. 50:6 (2014), 957-973.

[Bruggi and Taliercio 2015] M. Bruggi and A. Taliercio, "Analysis of no-tension structures under monotonic loading through an energy-based method", Comput. Struct. 159 (2015), 14-25.

[Bruggi and Taliercio 2017] M. Bruggi and A. Taliercio, "Optimal strengthening of no-tension structures with externally bonded reinforcing layers or ties”, Struct. Multidiscip. Optim. 55:5 (2017), 1831-1846. 
PROOFS - PAGE NUMBERS ARE TEMPORARY

116

DEBORAH BRICCOLA, MATTEO BRUGGI AND ALBERTO TALIERCIO

$1^{1} / 2 \frac{1}{2}$

[Como 2016] M. Como, Statics of historic masonry constructions, Springer Series Solid Struct. Mech. 5, Springer, 2016.

[Cowin and Mehrabadi 1995] S. C. Cowin and M. M. Mehrabadi, "Anisotropic symmetries of linear elasticity", Appl. Mech. Rev. (ASME) 48:5 (1995), 247-285.

4 [Cuomo and Ventura 2000] M. Cuomo and G. Ventura, "A complementary energy formulation of no tension masonry-like solids", Comput. Methods Appl. Mech. Eng. 189:1 (2000), 313-339.

6 [Del Piero 1989] G. Del Piero, "Constitutive equation and compatibility of the external loads for linear elastic masonry-like materials", Meccanica (Milano) 24:3 (1989), 150-162.

- [Di Carlo et al. 2017] F. Di Carlo, S. Coccia, and M. Como, "Rocking in presence of cracking of masonry wall piers", Key Eng. Mater. 747 (2017), 678-685.

9 [Fortunato et al. 2016] A. Fortunato, E. Babilio, M. Lippiello, A. Gesualdo, and M. Angelillo, "Limit analysis for unilateral masonry-like structures", Open Const. Build. Tech. J. 10:suppl. 2:M12 (2016), 346-362.

1 [Fortunato et al. 2018] A. Fortunato, F. Fabbrocino, M. Angelillo, and F. Fraternali, "Limit analysis of masonry structures with free discontinuities", Meccanica (Milano) 53:7 (2018), 1793-1802.

[Fuschi et al. 1995] P. Fuschi, G. Giambanco, and S. Rizzo, "Nonlinear finite element analysis of no-tension masonry structures", Meccanica (Milano) 30:3 (1995), 233-249.

[Heyman 1966] J. Heyman, “The stone skeleton”, Int. J. Solids Struct. 2:2 (1966), 249-279.

[Lourenço 2001] P. B. Lourenço, "Analysis of historical constructions: from thrust-lines to advanced simulations", pp. 91-116 in Historical constructions (Guimarães, 2001), edited by P. B. Lourenço and P. Roca, Univ. Minho, Guimarães, 2001.

[Lourenço et al. 1997] P. B. Lourenço, R. de Borst, and J. G. Rots, "A plane stress softening plasticity model for orthotropic materials", Int. J. Numer. Methods Eng. 40:21 (1997), 4033-4057.

[Maier and Nappi 1990] G. Maier and A. Nappi, “A theory of no-tension discretized structural systems", Eng. Struct. 12:4 $20^{1} / 2 \frac{20}{21}$ (1990), 227-234.

21 [Marfia and Sacco 2005] S. Marfia and E. Sacco, "Numerical procedure for elasto-plastic no-tension model", Int. J. Comput. Methods Eng. Sci. Mech. 6:3 (2005), 187-199.

[Mastrodicasa 1943] S. Mastrodicasa, Dissesti statici delle strutture edilizie, Hoepli, Milano, 1943.

[Mehrabadi and Cowin 1990] M. M. Mehrabadi and S. C. Cowin, "Eigentensors of linear anisotropic elastic materials", Quart. J. Mech. Appl. Math. 43:1 (1990), 15-41.

[Papa and Taliercio 2005] E. Papa and A. Taliercio, "A visco-damage model for brittle materials under monotonic and sustained stresses", Int. J. Numer. Anal. Methods Geomech. 29:3 (2005), 287-310.

[Pelà et al. 2011] L. Pelà, M. Cervera, and P. Roca, "Continuum damage model for orthotropic materials: application to masonry", Comput. Methods Appl. Mech. Eng. 200:9-12 (2011), 917-930.

[Rovati and Taliercio 2003] M. Rovati and A. Taliercio, "Stationarity of the strain energy density for some classes of anisotropic solids", Int. J. Solids Struct. 40:22 (2003), 6043-6075.

[Svanberg 1987] K. Svanberg, "The method of moving asymptotes: a new method for structural optimization", Int. J. Numer. Methods Eng. 24:2 (1987), 359-373.

Received 29 Apr 2018. Revised 25 Jul 2018. Accepted 3 Dec 2018.

DEBORAH BRICCOLA: deborah.briccola@polimi.it

Department of Civil and Environmental Engineering, Politecnico di Milano, Milano, Italy

Matteo BRUGGi: matteo.bruggi@polimi.it

Department of Civil and Environmental Engineering, Politecnico di Milano, Milano, Italy

AlBERTO TALIERCIO: alberto.taliercio@polimi.it

$39^{1} / 2 \frac{39}{40}$ Department of Civil and Environmental Engineering, Politecnico di Milano, Milano, Italy

41

42

mathematical sciences publishers 